\title{
MU-MIMO Downlink Capacity Analysis and Optimum Code Weight Vector Design for 5G Big Data Massive Antenna Millimeter Wave Communication
}

\author{
Adam Mohamed Ahmed Abdo, ${ }^{1}$ Xiongwen Zhao $\left(\mathbb{D},{ }^{1}\right.$ Rui Zhang, ${ }^{2}$ Zhenyu Zhou $\left(\mathbb{D},{ }^{1}\right.$ \\ Jianhua Zhang $\left(\bullet,{ }^{3}\right.$ Yu Zhang, ${ }^{1}$ and Imran Memon $\left(\oplus^{4}\right.$ \\ ${ }^{1}$ School of Electrical and Electronic Engineering, North China Electric Power University, Beijing 102206, China \\ ${ }^{2}$ National Key Laboratory of Electromagnetic Environment, China Research Institute of Radiowave Propagation, \\ Qingdao 266107, China \\ ${ }^{3}$ School of Information and Communication Engineering, Beijing University of Posts and Telecommunications, \\ Beijing 100876, China \\ ${ }^{4}$ College of Computer Science, Zhejiang University, Hangzhou, Zhejiang 310027, China
}

Correspondence should be addressed to Xiongwen Zhao; zhaoxw@ncepu.edu.cn

Received 8 January 2018; Revised 1 April 2018; Accepted 18 April 2018; Published 22 May 2018

Academic Editor: Javier Prieto

Copyright (C) 2018 Adam Mohamed Ahmed Abdo et al. This is an open access article distributed under the Creative Commons Attribution License, which permits unrestricted use, distribution, and reproduction in any medium, provided the original work is properly cited.

\begin{abstract}
Multiuser multiple input multiple output (MU-MIMO) wireless communication system provides substantial downlink throughput in millimeter wave (mmWave) communication by allowing multiple users to communicate at the same frequency and time slots. However, the design of the optimum beam-vector for each user to minimise interference from other users is challenging. In this paper, based on the concept of signal-to-leakage plus noise ratio (SLNR), we analyze the ergodic sum-rate capacity using statistical Eigen-mode (SE) and zero-forcing (ZF) models with Ricean fading channel. In the analysis, the orthogonality of channel vectors between users is assumed to guarantee interference cancelation from other cochannel users. The impact of the number of antenna elements on the achievable sum-rate capacity obtained by dirty paper coding (DPC) method considered as a nonlinear scheme for approximating average system capacity is studied. A power iterative precoding scheme that iteratively finds the most dominant eigenvector (optimum weight vector) for minimising cochannel interference (CCI), that is, maximising the SLNR for all users simultaneously, is designed resulting in enhancement of average system capacity. The average system capacities achieved by the proposed power iterative technique in this study compared with the singular value decomposition (SVD) method are in the ranges of 5-11 bps/Hz and 1-6 bps/Hz, respectively. Therefore, the proposed power iterative method achieves higher performance than the SVD regarding achievable sum-rate capacity.
\end{abstract}

\section{Introduction}

Millimeter wave (mmWave) communication which explores shorter propagation distance in frequency band of $20-40 \mathrm{GHz}$ is a key enabler for the fifth generation $(5 \mathrm{G})$ mobile communication systems [1]. The mmWave communication also provides significant benefits to a variety of applications such as vehicular communication, wire-able networks, and autonomous robots [2]. In downlink transmission for a MU-MIMO system, a base station (BS) serves multiple users simultaneously in the same frequency and time slot. Thus the throughput can be enhanced by spatial multiplexing. However, the cochannel interference (CCI) becomes a dominant factor in capacity due to nonorthogonal signalling [3]. Hence, minimising CCI as much as possible at the end users is of importance. CCI can be suppressed by using linear precoders and decoders at transmitter and receiver sides [4]. Additionally, the channel state information (CSI) as well as partial information on the transmitter side can be used to improve the system's performance [5]. For all 
users served by the BS, the CSI should be known in advance at the BS to support the CCI minimisation which is not available at user's end. Explicitly, the overhead on the system is reduced in this approach because the channel information feedback is not required $[6,7]$. To perfectly cancel CCI at each end user, the restriction on the system configuration is necessary. For instance, the number of antenna elements at the BS should be larger than the total number of antennas at the end users [7]. This assumption is usually valid for single antenna per user which is generally adopted in the MU-MIMO system.

There are several studies that have been undertaken in MU-MIMO downlink systems on how to minimise the CCI problem related to CSI and analyze the achievable sum-rate per user as well as the average system capacity. In [8], an in-depth capacity analysis for nonorthogonal multiple access (NOMA) mmWave massive MIMO systems was provided. A simplified mmWave channel model was also explored by extending the uniform random single-path (UR-SP) model with the angle-of-arrival (AOA). Furthermore, the capacity analysis was divided into high and low signal to noise ratio (SNR) regimes, where the dominant factors for the signal to interference plus noise ratio (SINR) were determined as interference and noise. A multicast beamforming approach was proposed in [9], where users located within the proximity of the BS can receive two different data streams simultaneously, while those away can receive only one data stream. In [7], a model was designed to transmit beamforming vectors to maximise the signal to leakage ratio (SLR), minimising transmission power which may cause interference to other users. The model is not restricted to a certain number of transmission antenna elements and can be extended to more general scenarios. An asymptotic deterministic SLNR optimisation approach for regularized zero-forcing (RZF) considering perfect CSI and antenna correlation was proposed [10]. It was found that when the users are homogeneously distributed and the number of antenna elements is large enough $(M \rightarrow \infty)$, the SLNR is asymptotically equal to SINR. In [11], an efficient statistical Eigen-mode space division multiple access (SE-SDMA) scheme for downlink ergodic sum-rate analysis based on SLNR was proposed. To maximise the approximate ergodic sum-rate capacity, authors in [12] designed an optimal beamforming vector for each user based on a three-dimensional beamforming algorithm. The authors in [3] focused on imperfect CSI scenario and developed a robust SLNR approach for compensating performance degradation caused by random CSI errors. In [13], a precoding scheme for heterogeneous networks (Het-Nets) was proposed based on SLNR under imperfect CSI scenario. In this case, regularization parameter was used to "weight" the precoding information for other cells (BSs).

In this paper based on the SLNR concept and Ricean fading channel model with perfect CSI in MU-MIMO systems, the statistical Eigen-mode (SE) and zero-forcing (ZF) models are derived. In addition, the average achievable sumrate by each user and the overall average system capacity with different number of users are analyzed. Besides, the expression of dirty paper coding (DPC) method as a function of number of users $(U)$ and number of antenna elements $(M)$ is addressed. Moreover, the impact of $U$ on the average achievable sum-rate is demonstrated. The Ricean fading channel with the line-of-sight (LOS) and scattering components as channel model are adopted. We find that the ergodic capacity of DPC is approximately free from channel matrix which mainly depends on the number of BS antenna elements and average transmission power.

Furthermore, by exploring the leakage signal criteria, we propose a new design solution for precoding based on the proposed power iteration technique. The technique finds the optimum weight vector which maximises the SLNR, that is, enhances the system capacity by obtaining the dominant eigenvector for minimising the CCI. Finally, we compare the proposed method with the conventional solutions such as SVD. The numerical results demonstrate that significant throughput performance can be achieved with the proposed technique.

A comparative study of the proposed and SVD methods regarding computation complexity and storage is also carried out. The simplicity of the proposed algorithm is observed in approximating only one eigenvalue of a matrix in a sequence which is considerably more efficient as the number of iterations increase. In comparison, the SVD has to calculate all eigenvectors and pick the one with the most significant eigenvalue (the maximum eigenvalue), which requires larger computation capability and storage capacity.

The contributions of this paper are in twofold:

(1) The SE and ZF beam former models in Ricean fading channel are examined based on SLNR instead of the commonly used SINR and orthogonal condition of the beam weight vector in MU-MIMO systems. The linear achievable sum-rate capacity is investigated using these models to validate the proposed method. Moreover, in nonlinear achievable sum-rate capacity, the DPC technique regarding the impact of the number of users and antenna elements on the ergodic capacity is addressed. The results show that the number of antenna elements has a significant contribution to the ergodic capacity, and the DPC sum-rate capacity logarithmically increases with the number of users.

(2) A new optimum weight vector is developed based on the proposed power iteration method, which allows each user to maximise the SLNR and minimise the CCI from other users. The proposed method compared with the conventional SVD method shows that the proposed method can achieve better performance with relatively low cost.

This paper is organized as follows. In Section 2, the MUMIMO downlink system is introduced. The beamforming and leakage signal approaches are presented in Section 3. In Section 4, the results and discussion of the study are presented. Finally, the conclusion is drawn in Section 5. The significant proofs of the algorithm are addressed in the appendix.

In this paper, the superscript $(\bullet)^{H},(\bullet)^{T}$ and $(\bullet)^{*}$ denote the conjugate-transpose (Hermitian), transpose, and conjugate, respectively. $E\{\bullet\}, \mathbf{I}_{M}$, and $C N$ are the expectation operator, square identity matrix of size $M \times M$, and Gaussian random complex numbers, respectively. 


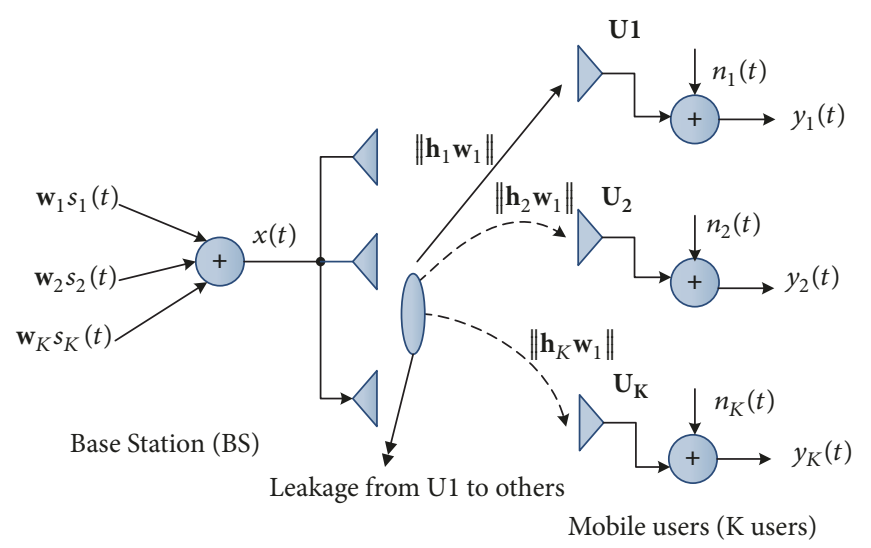

FIGURE 1: Schematic diagram for leakage signals in MU-MIMO system model from the first user to all other users.

\section{System Model}

Consider the MU-MIMO system model shown in Figure 1. The BS is equipped with $M$ uniform linear transmission antennas that serve $U$ users simultaneously. Assume that each user is equipped with a single antenna element [3]. Additionally, the number of the BS antenna elements is assumed to be larger than that of users $(M \geq U)$; and the power is equally allocated among all users [11, 12].

2.1. Signal Model. From the previous assumptions, the received signal $y_{k}$ at $k$ th user is given by

$$
y_{k}=\sqrt{p_{k}} \mathbf{h}_{k}^{H} \mathbf{w}_{k} x_{k}+\sqrt{p_{j}} \sum_{j=1, j \neq k}^{U} \mathbf{h}_{k}^{H} \mathbf{w}_{j} s_{j}+n_{k} .
$$

The expression in (1) can be rewritten in a more simplified form as

$$
y_{k}=\sqrt{p_{k}} \sum_{j=1}^{U} \mathbf{h}_{k}^{H} \mathbf{w}_{j} s_{j}+n_{k}
$$

where $\mathbf{h}_{k} \in \mathbb{C}^{1 \times M}$ is the flat Ricean downlink channel vector between the BS and the $k$ th user. The weight vector $\mathbf{w}_{j} \in \mathbb{C}^{M \times 1}$ is unit normalised beamforming vector of user $j$ and satisfies $\left\|\mathbf{w}_{j}\right\|=1$. Furthermore, the vector $s_{j}$ is the transmitted data symbol of user $j$ with $E\left[\left|s_{j}\right|^{2}\right]=1 . n_{k} \rightarrow$ $C N\left(0, \sigma_{k}\right)$ is unit normalised $\left(\sigma_{k}^{2}=1\right)$ complex additive white Gaussian noise (AWGN). Moreover, $p_{k}$ is the transmission power of the $k$ th user under the constraint $\sum_{k=1}^{U} p_{k} \leq P$. Since the total transmission power $(P)$ is divided among all the users equally, the average power can be written as $p_{k}=P / U$ for each user $[3,11,12]$. Consequently, the signal magnitude of each user can be written as $p\left|\mathbf{h}_{k}^{H} \mathbf{w}_{k}\right|$ while the interference of the $k$ th user is determined by the sum of interference from all other users. Hence, the interference can be denoted as $\sum_{j=1, j \neq k}^{U} p\left|\mathbf{h}_{k}^{H} \mathbf{w}_{j}\right|^{2}$. Thus the SINR of the $k$ th user can be expressed as in (3) [12, 14]:
(Note: for simplicity we drop the "subscript $k$ " from $p_{k}$ in the rest of the paper).

$$
\operatorname{SINR}_{k}=\frac{p\left|\mathbf{h}_{k}^{H} \mathbf{w}_{k}\right|^{2}}{1+\sum_{j=1, j \neq k}^{U} p\left|\mathbf{h}_{k}^{H} \mathbf{w}_{j}\right|^{2}} .
$$

The corresponding average achievable data rate for user $k$ can be written as

$$
R_{k}=E\left[\log _{2}\left(1+\operatorname{SINR}_{k}\right)\right] .
$$

By substituting (3) into (4), the average achievable sum rate per user is given as in $(5)[15,16]$.

$$
R_{k}=E\left\{\log _{2}\left(1+\frac{p\left|\mathbf{h}_{k}^{H} \mathbf{w}_{k}\right|^{2}}{1+\sum_{j=1, j \neq k}^{U} p\left|\mathbf{h}_{k}^{H} \mathbf{w}_{j}\right|^{2}}\right)\right\} .
$$

Subsequently, the overall system ergodic sum-rate capacity can be written in $\mathrm{bps} / \mathrm{Hz}$ as

$$
R_{\text {system }}=\sum_{k=1}^{U} R_{k}
$$

2.2. Channel Model. In this subsection, we describe the channel model as in $[11,12]$. In the most recent studies on mmWave communication, the Rayleigh fading channel model is employed which faces difficulty in capturing the fading variation in the presence of the LOS component. Moreover, mmWave MU massive antenna system can be used mainly in $5 \mathrm{G}$ hotpot scenarios in the LOS case. To overcome the capturing problem in Rayleigh fading channel, the Ricean fading channel is applied in this paper. Considering the Ricean fading channel model, the channel vector has two components: LOS and Rayleigh random distribution components. Thus, the channel vector can be expressed as [11]

$$
\mathbf{h}_{k}=\sqrt{\frac{K_{k}}{K_{k}+1}} \overline{\mathbf{h}}_{k}+\sqrt{\frac{1}{K_{k}+1}} \mathbf{h}_{w, k},
$$

where $K_{k}$ is the power ratio between the LOS component and scattering components, also called Ricean $K$-factor of the 
$k$ th user. Moreover, the channel vector $\mathbf{h}_{w, k}$ is the random component, and the entries are independent and identical distribution complex Gaussian random variables. Vector $\overline{\mathbf{h}}_{k}(k=1,2, \ldots, U)$ is the deterministic component of the channel mean vector of the $k$ th user. Based on the assumption that the BS is equipped with $M$ uniform linear array antennas, the deterministic channel vector for each user can be written as $[17,18]$

$$
\overline{\mathbf{h}}_{k}=\left[1, e^{j 2 \pi \lambda^{-1} d \sin \left(\theta_{k}\right)} \cdots e^{j(M-1) 2 \pi \lambda^{-1} d \sin \left(\theta_{k}\right)}\right],
$$

where $d$ is the space between two antenna elements, $\lambda$ is the wavelength of the signal, and $\theta_{k}$ is the angle of departure (AoD) for the $k$ th user, $\theta_{k} \in[-\pi, \pi]$.

\section{Beamforming and Leakage Signals}

It is assumed that all users have a perfect instantaneous knowledge of their channel vectors while the BS knows all users channel vector including the channel mean vector. Additionally, assuming $U$ users, it is difficult to maximise the average system capacity due to a couple of $U$ calculations (capacity of each user) required. Therefore, it is hard to use SINR directly to obtain the optimum beamforming vector $\left(\mathbf{w}_{k}\right)$. To overcome this problem, the SLNR can be easily implemented to control the leakage signals from a specific user to other users as shown in Figure 1. The SLNR of the $k$ th user can be written as in (9) by assuming unit normalised Gaussian noise $[4,16]$ :

$$
\operatorname{SLNR}_{k}=\frac{p\left|\mathbf{h}_{k}^{H} \mathbf{w}_{k}\right|^{2}}{1+p \sum_{j=1, j \neq k}^{U}\left|\mathbf{h}_{j}^{H} \mathbf{w}_{k}\right|^{2}} .
$$

Equations (3) and (9) have different denominators to define SINR and SLNR. In (3), the user channel vector $\mathbf{h}_{k}$ is used with other users' beamforming vectors $\left.\mathbf{w}_{j}\right|_{j=1} ^{U}, j \neq k$ to calculate SLNR of the $k$ th user. While in (9) the $k$ th user beamforming vector $\mathbf{w}_{k}$ is used with other users' channel vectors $\left.\mathbf{h}_{j}\right|_{j=1} ^{U}, j \neq k$ to calculate SLNR of the $k$ th user. Besides, the lower bound (LB) on the average SLNR of the $k$ th user can be represented as $E\left\{\mathrm{SLNR}_{k}\right\} \geq\left\{\mathrm{SLNR}_{k}\right\}_{\mathrm{LB}}$ demonstrated in $[11,16]$ :

$$
\begin{aligned}
\operatorname{SLNR}_{k} & =\left\{\mathrm{SLNR}_{k}\right\}_{\mathrm{LB}} \\
& =\frac{p \mathbf{w}_{k}^{H} \mathbf{h}_{k}^{H} \mathbf{h}_{k} \mathbf{w}_{k}}{1+p \sum_{j=1, j \neq k}^{U} \mathbf{w}_{k}^{H} \mathbf{h}_{j}^{H} \mathbf{h}_{j} \mathbf{w}_{k}} \\
\left\{\mathrm{SLNR}_{k}\right\}_{\mathrm{LB}} & \triangleq \frac{p \mathbf{w}_{k}^{H} \mathbf{R}_{k} \mathbf{w}_{k}}{1+p \mathbf{w}_{k}^{H} \sum_{j=1, j \neq k}^{U} \mathbf{R}_{j} \mathbf{w}_{k}} .
\end{aligned}
$$

In (10), we can have $\mathbf{R}_{k} \triangleq E\left\{\mathbf{h}_{k}^{H} \mathbf{h}_{k}\right\}$ :

$$
\mathbf{R}_{j} \triangleq E\left\{\mathbf{h}_{j}^{H} \mathbf{h}_{j}\right\}=\frac{K_{j}}{K_{j}+1} \overline{\mathbf{R}}_{j}+\frac{1}{K_{j}+1} \mathbf{I}_{M}
$$

where $\mathbf{R}_{k}$ is a Hermitian matrix (channel correlation matrix) which can be constructed as in (12), where $\overline{\mathbf{R}}_{j} \triangleq \overline{\mathbf{h}}_{j}^{H} \overline{\mathbf{h}}_{j}$, and $\overline{\mathbf{h}}_{j}$ is calculated in (8). Hence, $\mathbf{R}_{k}$ of the $k$ th user can be decomposed regarding the deterministic component of channel vector $\overline{\mathbf{h}}_{k}$ after normalisation by a factor $\sqrt{M}$ [6]. Therefore, we can use the normalised version $\overline{\mathbf{h}}_{k} / \sqrt{M}$ in the decomposition of the channel correlation matrix as in

$$
\mathbf{R}_{k}=\left(\begin{array}{cc}
\frac{\overline{\mathbf{h}}_{k}^{H}}{\sqrt{M}} \widetilde{\mathbf{U}}_{k}^{H}
\end{array}\right)\left(\begin{array}{cccc}
\frac{K_{k} M}{K_{k}+1}+\frac{1}{K_{k}+1} & 0 & \cdots & 0 \\
0 & \frac{1}{K_{k}+1} & \cdots & 0 \\
\vdots & \vdots & \ddots & \vdots \\
0 & 0 & \cdots & \frac{1}{K_{k}+1}
\end{array}\right)\left(\begin{array}{c}
\overline{\mathbf{h}}_{k}^{H} \\
\sqrt{M} \\
\widetilde{\mathbf{U}}_{k}
\end{array}\right) \text {, }
$$

$$
\begin{aligned}
& \mathbf{w}_{k}^{H} \mathbf{R}_{k} \mathbf{w}_{k}=\frac{K_{k}}{K_{k}+1} M+\frac{1}{K_{k}+1} \\
& \mathbf{w}_{k}^{H} \mathbf{R}_{j} \mathbf{w}_{k}=\frac{1}{K_{k}+1}, \quad \text { for } j \neq k .
\end{aligned}
$$

The maximum value of the lower bound can be achieved if $\mathbf{w}_{k}^{\text {opt }}$ is orthogonal to $(1 / \sqrt{M}) \overline{\mathbf{h}}_{j}^{H}$. By substituting (15) in (10), we have

$$
\left\{\mathrm{SLNR}_{k}\right\}_{\mathrm{LB}}^{\max }=\frac{p\left(\left(K_{k} /\left(K_{k}+1\right)\right) M+1 /\left(K_{k}+1\right)\right)}{1+p \sum_{j=1, j \neq k}^{U}\left(1 /\left(K_{j}+1\right)\right)}
$$

$$
\begin{aligned}
& \qquad \overline{\mathbf{h}}_{j} \mathbf{w}_{k}^{\text {opt }}=\frac{1}{\sqrt{M}} \overline{\mathbf{h}}_{j} \overline{\mathbf{h}}_{k}^{H}=0, \quad j \neq k, j=1,2, \ldots, U, \\
& \text { where vector } \mathbf{w}_{k}^{\text {opt }} \text { is orthogonal to }(1 / \sqrt{M}) \overline{\mathbf{h}}_{j}^{H} \text {. Accordingly, } \\
& \text { from (12) we have }
\end{aligned}
$$

On the other hand, to minimise the denominator of (9) we have

where $\widetilde{\mathbf{U}}_{k}^{H}$ is the orthogonal subspace of $\overline{\mathbf{h}}_{k}^{H} / \sqrt{M}$. Then we can obtain the optimum beamforming vector which maximises the lower bound of the SLNR as follows:

$$
\mathbf{w}_{k}^{\text {opt }}=\frac{1}{\sqrt{M}} \overline{\mathbf{h}}_{k}^{H} \quad \text { for } k=1,2, \ldots, U .
$$


In (16), the lower bound is directly affected by the number of antenna elements $M$, the Ricean factor $K$, and the number of users $U$. Assuming that the number of users is increased as $k=1 \rightarrow U$, taking $K=0$ and $K \rightarrow \infty$, the lower bounds of SLNR are expressed approximately as $\left.(p /(1+p(U-1)))\right|_{K=0}$ and $\left.M * p\right|_{K \rightarrow \infty}$, respectively. Therefore, it is seen that the lower bound is independent of $M$ when $K=0$.

3.1. Analysis of Linear Achievable Rates by SE and ZF. In this section, we evaluate the Ergodic sum-rate capacity of SE obtained by (13) and (14) and assume that ZF capacity has perfect CSI when calculating the mean gap loss between $R^{\mathrm{SE}}$ and $R^{\mathrm{ZF}}$. Based on the orthogonal beamforming condition in (13) and (14), the achievable sum-rate capacity obtained by the $k$ th user is given by

$$
\begin{aligned}
R_{k}^{\mathrm{SE}} & =E\left\{\log _{2}(1\right. \\
& \left.\left.+\frac{p\left|(1 / \sqrt{M}) \mathbf{h}_{k} \overline{\mathbf{h}}_{k}^{H}\right|^{2}}{1+p \sum_{j=1, j \neq k}^{U}\left|(1 / \sqrt{M}) \mathbf{h}_{k} \overline{\mathbf{h}}_{j}^{H}\right|^{2}}\right)\right\} \\
& \approx E\left\{\log _{2}\left(1+\frac{p\left|\mathbf{h}_{k} \mathbf{w}_{k}^{\mathrm{opt}}\right|^{2}}{1+p \sum_{j=1, j \neq k}^{U}\left|\mathbf{h}_{k} \mathbf{w}_{j}^{\mathrm{opt}}\right|^{2}}\right)\right\} .
\end{aligned}
$$

The achievable sum-rate capacity by ZF is expressed as

$$
R_{k}^{\mathrm{ZF}}=\log _{2}\left(1+p\left|\mathbf{h}_{k} \mathbf{w}_{k}^{\mathrm{ZF}}\right|\right)
$$

where $\mathbf{w}_{k}^{\mathrm{ZF}}$ is the unit normalised beamforming vector, which is selected as the $k$ th column of the normalised matrix $\mathbf{w}=\mathbf{H}\left(\mathbf{H H}^{H}\right)^{-1}$ with $\mathbf{H}=\left[\begin{array}{llll}\mathbf{h}_{1}^{H} & \mathbf{h}_{2}^{H} & \cdots & \mathbf{h}_{U}^{H}\end{array}\right]$ to suppress the interference from all other users for $\left|\mathbf{h}_{j} \mathbf{w}_{k}^{\mathrm{ZF}}\right|=0$, if $k \neq j$. Accordingly, the mean gap loss $\Delta R^{\mathrm{ZF}-\mathrm{SE}}$ with perfect instantaneous CSI is given by

$$
\Delta R^{\mathrm{ZF}-\mathrm{SE}}=R^{\mathrm{ZF}}-R^{\mathrm{SE}}
$$

From the orthogonal condition $(1 / \sqrt{M}) \overline{\mathbf{h}}_{j} \overline{\mathbf{h}}_{k}^{H}=\left.0\right|_{j \neq k}$ which is addressed in (14), we neglect the interference from the other users to the $k$ th user regarding the signal component. In this case the sum-rate capacity $R^{\mathrm{SE}}$ used in (14) changes to the loose bound sum-rate capacity of the $k$ th user expressed as [11]

$$
\begin{aligned}
R_{k}^{\mathrm{SE}} \geq & E\left\{\log _{2}\left(1+p\left|\frac{1}{\sqrt{M}} \mathbf{h}_{k} \overline{\mathbf{h}}_{k}^{H}\right|^{2}\right)\right\} \\
& -E\left\{\log _{2}\left(1+p \sum_{j=1, j \neq k}^{U}\left|\frac{1}{\sqrt{M}} \mathbf{h}_{k} \overline{\mathbf{h}}_{j}^{H}\right|^{2}\right)\right\} .
\end{aligned}
$$

Using (18) and (20), we rewrite (19) as the mean gap loss in (21) as

$$
\begin{aligned}
& \Delta R^{\mathrm{ZF}-\mathrm{SE}} \\
& \leq \sum_{k=1}^{U} E\left\{\log _{2}\left(1+p\left|\mathbf{h}_{k} \mathbf{w}_{k}^{\mathrm{ZF}}\right|^{2}\right)\right\} \\
& \quad-E\left\{\log _{2}\left(1+p\left|\frac{1}{\sqrt{M}} \mathbf{h}_{k} \overline{\mathbf{h}}_{k}^{H}\right|^{2}\right)\right\} \\
& +E\left\{\log _{2}\left(1+p \sum_{j=1, j \neq k}^{U}\left|\frac{1}{\sqrt{M}} \mathbf{h}_{k} \overline{\mathbf{h}}_{j}^{H}\right|^{2}\right)\right\} .
\end{aligned}
$$

3.2. Nonlinear Achievable Rate Analysis by DPC. The BS can serve all users simultaneously and achieve maximum system capacity as much as possible as the CSI is known at the BS. In MIMO systems, the system capacity obtained by downlink strategy is called DPC. Practically, it is challenging to implement DPC because the encoding and decoding have high computations which is ineffective for large number of users. The achievable sum-rate capacity of the DPC can be written as [19]

$$
\begin{array}{r}
R_{\mathrm{DPC}}=\max \log \left(\left|1+\sum_{k=1}^{U} p \mathbf{h}_{k}^{*} \mathbf{h}_{k}\right|\right), \\
\quad p \geq 0, \underset{k=1}{U} \leq P .
\end{array}
$$

When the number of users is large, the DPC achievable sumrate capacity is approximately expressed as $[19,20]$

$$
R_{\mathrm{DPC}}=M \log \left(1+\frac{P}{M} \log U\right) .
$$

From (23), we can observe that the sum-rate capacity increases linearly with the number of antenna elements. However, the approximate achievable sum-rate capacity is a nonlinear function of $U$. Therefore, the DPC is regarded as a nonlinear model.

3.3. Optimum Weight Vector Formulation. In this subsection, we find the solution for optimum weight vector $\mathbf{w}^{\mathrm{opt}}$, in which the maximum SLNR can be achieved to guarantee that all users have the ability to access the limited resources. For equal transmission of power to all users in a given case, we need to design $\mathbf{w}_{k}, k=1,2, \ldots, U$ to maximise the SLNR for each user at the same time minimise interference. Hence, the optimum weight vector $\mathbf{w}_{k}^{\text {opt }}=\arg \max \left(\operatorname{SLR}_{k}\right)$ can be written as follows:

$$
\begin{aligned}
\mathbf{w}_{k}^{\text {opt }} & =\arg \max \left(\frac{\left\|\mathbf{h}_{k} \mathbf{w}_{k}\right\|^{2}}{\sum_{j=1, j \neq k}^{U}\left\|\mathbf{h}_{j} \mathbf{w}_{k}\right\|^{2}}\right) \\
\mathrm{SLR}_{k} & =\frac{\left\|\mathbf{h}_{k} \mathbf{w}_{k}\right\|^{2}}{\sum_{j=1, j \neq k}^{U}\left\|\mathbf{h}_{j} \mathbf{w}_{k}\right\|^{2}} .
\end{aligned}
$$


Step 1: Initial Inputs. number of users $U$;

number of iteration $N$;

initial non-zero vector $\mathbf{X}_{0}$;

Tolerance Tol.

Step 2: For $k=1: U / /$ to construct the CICTM for each user;

Calculate $\mathbf{h}_{w, k}$ and $\overline{\mathbf{h}}_{k}$ as defined in Section 2.2;

Calculate for $\mathbf{h}_{k}$ as in (7);

End

Construct the CICTM $\widetilde{\mathbf{h}}_{k}$ for each user as in (29);

Step 3: For $k=1: U / /$ to compute $\mathbf{w}_{k}^{\text {opt }}$ for each user.

Calculate matrix $\mathbf{A}$ for $k$ th user as in (31)

Step 4: While $i \leq N$;

set: $\mathbf{y}_{i}=\mathbf{A} \mathbf{x}_{i-1}$, and $\mathbf{x}_{i}=\mathbf{y}_{i} / \alpha_{i} ;$ (normalisation)

"the value of $\mathbf{x}_{i}$ is the scaled version of $\mathbf{y}_{i}$ "

if $\left|\alpha_{i}-\alpha_{i-1}\right| \leq$ Tol, then $i=N+1$;

else, set $i=i+1$;

end

Step 5: calculate the dominant eigenvector $\mathbf{v}_{1} \approx \mathbf{x}_{i}$;

End;

the dominant eigenvalue $\lambda_{1}=\mathbf{x}_{i}^{*} \mathbf{A} \mathbf{x}_{i} / \mathbf{x}_{i}^{*} \mathbf{x}_{i}$;

Step 6: SLNR maximise weight vector $\widehat{\mathbf{w}}_{k}=\mathbf{x}_{i}$;

End;

Step 7: Output: the optimum weight vector $\widehat{\mathbf{w}}_{k}$ for each user.

Algorithm 1: Steps for the proposed power iteration method.

Equation (24) is subjected to

$$
\text { Constraint 1: }\left\|\mathbf{w}_{k}\right\|^{2}=1, \quad k=1,2, \ldots, U
$$

Constraint 2: $\left\|\mathbf{h}_{j} \mathbf{w}_{k}\right\|^{2}=0, \quad j, k=1,2, \ldots, U, j \neq k$.

Consequently, the SLNR is written as

$$
\operatorname{SLNR}_{k}=\frac{\left\|\mathbf{h}_{k} \mathbf{w}_{k}\right\|^{2}}{1+\sum_{j=1, j \neq k}^{U}\left\|\mathbf{h}_{j} \mathbf{w}_{k}\right\|^{2}} .
$$

Equation (27) is further written as

$$
\operatorname{SLNR}_{k}=\frac{\left\|\mathbf{h}_{k} \mathbf{w}_{k}\right\|^{2}}{1+\left\|\tilde{\mathbf{h}}_{k} \mathbf{w}_{k}\right\|^{2}},
$$

where $\widetilde{\mathbf{h}}_{k}$ is congregate interfering channel transfer matrix (CICTM) of the $k$ th user, which is an extended channel matrix that excludes $\mathbf{h}_{k}$ only for the $k$ th user. Then $\mathbf{h}_{k}$ is written as

$$
\begin{aligned}
& \tilde{\mathbf{h}}_{k}=\left[\begin{array}{llllllll}
\mathbf{h}_{1} & \mathbf{h}_{2} & \cdots & \mathbf{h}_{k-1} & \mathbf{h}_{k+1} & \cdots & \mathbf{h}_{U-1} & \mathbf{h}_{U}
\end{array}\right]^{T} \\
& \cdot\left(\sum_{\substack{j=1 \\
j \neq k}}^{U} 1 \times M\right),
\end{aligned}
$$

where $\widetilde{\mathbf{h}}_{k} \in \mathbb{C}^{M \times(U-1)}$ and $\mathbf{h}_{k} \in \mathbb{C}^{1 \times M}$. The optimum weight code vector can be derived from (28) as follows [21]:

$$
\mathbf{w}_{k}^{\text {opt }} \propto \max \text {. eigenvector }\left(\left(\mathbf{I}_{M}+\widetilde{\mathbf{h}}_{k}^{*} \widetilde{\mathbf{h}}_{k}\right)^{-1} \mathbf{h}_{k}^{*} \mathbf{h}_{k}\right),
$$

where $\mathbf{I}_{M}$ is $M \times M$ square identity matrix.
The optimum weight code vector in (30) is called SVD solution, which is determined from the matrix in (29). Sometimes, when calculating the maximum eigenvalues for the given matrix, the dominant eigenvector does not correspond to the optimum code weight vector. Hence, the eigenvalues are put in ascending or descending order; then an optimum vector corresponding to the most significant eigenvalue is easily selected but at the expense of extra overhead to the system [4, 7]. To enhance the system performance and minimise the cost, a new approximation method expected to improve the average user achievable sum-rate capacity is proposed.

3.4. Proposed Optimum Weight Vector Design. A new method is developed based on power iteration method to find the optimum weight vector. The method requires only one weight vector during implementation. Equation (30) is modified and adopted as the main matrix in the proposed power approximation method as follows:

$$
\mathbf{A}=\left(\mathbf{I}_{M}+\widetilde{\mathbf{h}}_{k}^{*} \widetilde{\mathbf{h}}_{k}\right)^{-1} \mathbf{h}_{k}^{*} \mathbf{h}_{k}
$$

3.4.1. Power Approximation Method. The power approximation method generates a sequence of vectors $\mathbf{A}^{i} \mathbf{x}_{0}$, where $\mathbf{x}_{0}$ is a nonzero initial selected vector. By normalising these sequence vectors under conditions stated in Section 3.4.3, the vector converges to the dominant eigenvector corresponding to the most significant eigenvalue. The normalisation is used to ensure that the most significant component of the given iteration is equal to one [22]. The steps in the proposed power iteration method are given in Algorithm 1. 
This algorithm is summarised in a sequence of iterations as follows:

$$
\mathbf{x}_{i}=\frac{1}{\alpha_{i}} \mathbf{A} \mathbf{x}_{i-1},
$$

where $\alpha_{i}$ is the component (magnitude) of vector $\mathbf{A x}_{i-1}$. Before proceeding to the next iteration, it is necessary to scale down (normalised) the sequence vectors in each approximation so as to keep the largest component at unity.

3.4.2. Convergence of the Algorithm. The matrix $\mathbf{A}$ is $n \times n$ square matrix with $n$ eigenvalues $\left[\lambda_{1}, \lambda_{2}, \lambda_{3}, \ldots, \lambda_{n}\right]$, which are in descending order as $\left|\lambda_{1}\right|>\left|\lambda_{2}\right| \geq\left|\lambda_{3}\right| \cdots \geq\left|\lambda_{n}\right|$. With the initial vector $\mathbf{X}_{0}$ chosen, the sequences $\left\{\mathbf{X}_{k}=\right.$ $\left.\left[\begin{array}{llll}x_{1}^{(k)} & x_{2}^{(k)} & \cdots & x_{n}^{(k)}\end{array}\right]^{T}\right\}$ and $\left\{c_{k}\right\}$ are recursively generated by the relations $\mathbf{Y}_{k}=\mathbf{A X}_{k}$ and $\mathbf{X}_{k+1}=\left(1 / c_{k+1}\right) \mathbf{Y}_{k}$, where $c_{k+1}=x_{j}^{(k)}$ and $x_{j}^{(k)}=\max _{1 \leq i \leq n}\left\{\left|x_{i}^{(k)}\right|\right\}$. These sequences converge to the dominant eigenvector $\mathbf{V}_{1}$ and eigenvalue $\lambda_{1}$, respectively, as $\lim _{k \rightarrow \infty} \mathbf{X}_{k}=\mathbf{V}_{1}$ and $\lim _{k \rightarrow \infty} c_{k}=\lambda_{1}$. The proof of convergence is referred to in Appendix A.

3.4.3. The Speed of Convergence. Referring to (A.6) in Appendix A, we observe that the coefficient of $\mathbf{V}_{j}$ in the sequence $\mathbf{X}_{k}$ goes to zero, which is proportional to $\left(\lambda_{\mathrm{j}} / \lambda_{1}\right)^{k}$; the convergence speed of sequence $\left\{\mathbf{X}_{k}\right\}$ to $\mathbf{V}_{1}$ is governed by $\left(\lambda_{2} / \lambda_{1}\right)^{k}$. Therefore, the rate of convergence and the convergence of the constants $\left\{c_{k}\right\}$ to $\lambda_{1}$ are linear. For any linear convergent sequence $\left\{p_{k}\right\}$, we can use the Aitken $\Delta^{2}$ technique which is used to make the linearly convergent sequences fast. The new convergence of the sequence $\left\{p_{k}\right\}$ can be written as

$$
\widehat{p}_{k}=\frac{\left(p_{k+1}-p_{k}\right)^{2}}{p_{k+2}-2 p_{k+1}+p_{k}}
$$

The convergence property of the algorithm is given by the following.

Theorem 1. Assume that there is one and only one eigenvalue $\lambda_{1}$ of $\mathbf{A}$, and $\lambda_{1}$ is semisimple; then either the initial vector $\mathbf{x}_{0}$ which has no component in the invariant subspace associated with $\lambda_{1}$ or the sequence of vectors generated by the algorithm converges to the eigenvector associated with $\lambda_{1}$ and $i$ and converges to $\lambda_{1}$. The proof is shown in Appendix $B$.

Definition 2. Eigenvalue $\lambda_{1}$ of matrix $\mathbf{A}$ is semisimple with the degree of $n$ if it has a geometric multiplicity one and algebraic multiplicity $n$. The vector $\mathbf{A}^{i} \mathbf{x}_{0}$ is normalised by a specific scalar $\alpha_{i}$ to make the most significant component of the vector unity. The initial vector $\mathbf{x}_{0}$ is decomposed as follows:

$$
x_{0}=\sum_{j=1}^{p} P_{j} \mathbf{x}_{0}
$$

where $P_{j}, j=1,2, \ldots, p$, are the spectral projectors associated with the eigenvalues $\lambda_{j}, j=1,2, \ldots, p$.
From the formula $\mathbf{A} P_{j}=P_{j}\left(\lambda_{j} \mathbf{I}+\mathbf{D}_{j}\right)$, the power can be written as $\mathbf{A}^{i} P_{j}=P_{j}\left(\lambda_{j} \mathbf{I}+\mathbf{D}_{j}\right)^{i}$, where $\mathbf{I}$ is the identity matrix and $\mathbf{D}_{j}$ is the diagonal matrix. Consequently, we have

$$
\begin{aligned}
\mathbf{x}_{i} & =\frac{1}{\alpha_{i}} \mathbf{A}^{i} \sum_{j=1}^{p} P_{j} \mathbf{x}_{0}=\frac{1}{\alpha_{i}} \sum_{j=1}^{p} \mathbf{A}^{i} P_{j} \mathbf{x}_{0} \\
& =\frac{1}{\alpha_{i}} \sum_{j=1}^{p} P_{j}\left(\lambda_{j} \mathbf{I}+\mathbf{D}_{j}\right)^{i} \mathbf{x}_{0} .
\end{aligned}
$$

Referring to the Definition 2, $\mathbf{D}_{1}=0$ because $\lambda_{1}$ is a semisimple eigenvalue. Thus, we obtain

$$
\begin{aligned}
\mathbf{x}_{i} & =\frac{1}{\alpha_{i}} \sum_{j=1}^{p} P_{j}\left(\lambda_{j} P_{j}+\mathbf{D}_{j}\right)^{i} \mathbf{x}_{0} \\
& =\frac{1}{\alpha_{i}}\left(\lambda_{1}^{k} P_{1} \mathbf{x}_{0}+\sum_{j=2}^{p} P_{j}\left(\lambda_{j} P_{j}+\mathbf{D}_{j}\right)^{i} \mathbf{x}_{0}\right) \\
& =\frac{\lambda_{1}^{i}}{\alpha_{i}}\left(P_{1} \mathbf{x}_{0}+\sum_{j=2}^{p} \frac{1}{\lambda_{1}^{i}}\left(\lambda_{j} P_{j}+\mathbf{D}_{j}\right)^{i} P_{j} \mathbf{x}_{0}\right) .
\end{aligned}
$$

The spectral radius of each operator $\left(\lambda_{j} P_{j}+\mathbf{D}_{j}\right) \lambda^{-1}<1$ and $\left|\lambda_{j} \lambda_{1}^{-1}\right|<1$ means that the $i$ th power will converge to zero. Theorem 1 is true when $P_{1} \mathbf{x}_{0}=0$. However, if $P_{1} \mathbf{x}_{0} \neq 0$, $\mathbf{x}_{i}$ converge to normalise $P_{1} \mathbf{x}_{0}$ so that the most significant component is one. Meanwhile, the scalar $\alpha_{i}$ converges to the eigenvalue $\lambda_{1}$, which is an immediate consequence in the form

$$
\mathbf{A x}_{i-1}=\alpha_{i} \mathbf{x}_{i}
$$

Thus, the sequence of vectors $\mathbf{x}_{i}$ is proven to converge to the optimum weight vector which maximises the SLNR in (28). The optimum weight vector is equal to the vector $\mathbf{x}_{i}$ :

$$
\widehat{\mathbf{w}}=\mathbf{x}_{i} \text {. }
$$

The result in (38), which is the main objective of this paper, maximises the $k$ th user SLNR when substituted in (28) to find $\mathrm{SLNR}_{k}$.

3.5. Cost Analysis. In this subsection, the cost function is analyzed regarding storage space required for the proposed power iteration technique and SVD method. Referring to (30), the value $\left(\mathbf{I}_{M}+\widetilde{\mathbf{h}}_{k}^{*} \widetilde{\mathbf{h}}_{k}\right)^{-1} \mathbf{h}_{k}^{*} \mathbf{h}_{k}$ results in a new square matrix with dimension $M \times M$, where $M$ denotes the number of antenna elements. Therefore, finding $\mathbf{w}_{k}^{\text {opt }}$ for each user by using the solution of SVD in (30), we need to find at least two matrices, $M \times M$ left eigenvectors matrix, $M \times M$ right eigenvectors matrix, and $M$ diagonal eigenvalues from the original $M \times M$ matrix. On the other hand, the required storage memory for the $k$ th user is equal to the size of three matrices in addition to the original square matrix. Hence, the total storage becomes $\left(3 M^{2}+M\right) * U$ (full SVD), where $U$ is the number of users. Then, the determination of the 


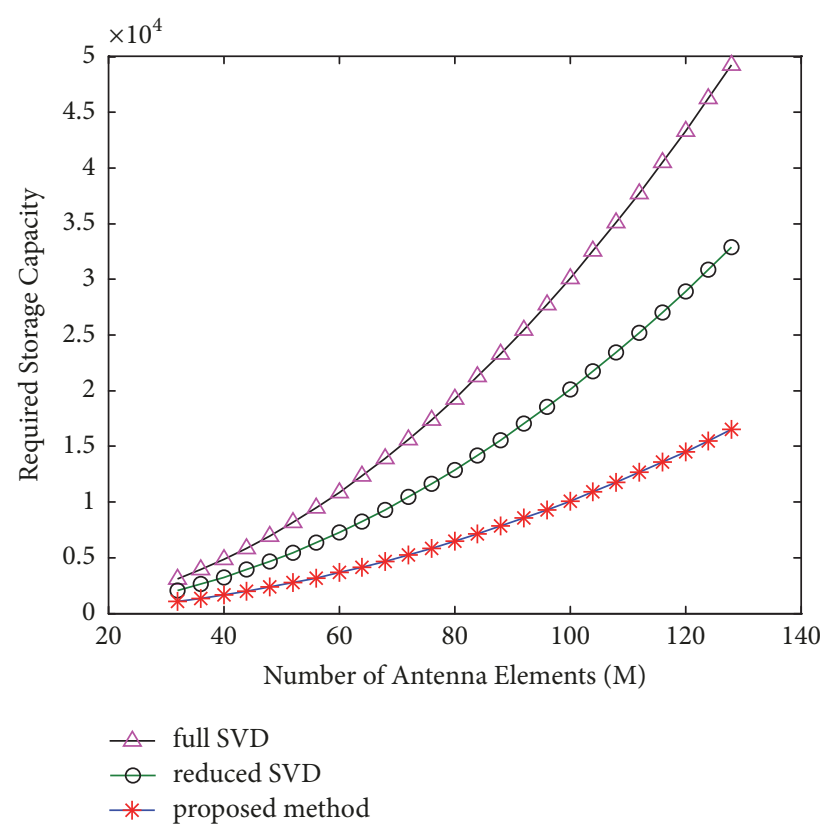

FIGURE 2: Storage capacity required by the proposed technique and the SVD (full SVD and reduced SVD) method regarding the number of antenna elements.

optimum weight vector can be from the right eigenvector or left eigenvector based on the corresponding maximum eigenvalue. Furthermore, we can reduce the size by taking only one side eigenvectors matrix (left or right) to save $M^{2}$ redundant storage size. Thus the reduced new storage capacity can be written as $\left(2 M^{2}+M\right) * U$ (reduced SVD).

Similarly, only the original square matrix with dimension $M \times M$ and a vector with size $M \times 1$ is required in the proposed power approximation method. In this case, there is no need for storing the previous iteration result as it can be overwritten up to the last iteration or reaches the determined tolerance. Thus, the required storage for the power iteration technique is $\left(M^{2}+M\right) * U$ which is much less than full and reduced SVDs.

Figure 2 shows the storage capacity required in the proposed power iteration technique and SVD method. From Figure 2, it is observed that the number of antenna elements has a significant effect on the cost function. However, the proposed method has much less storage space compared to SVD hence recommended for a massive MIMO with large antennas.

\section{Simulation Results and Discussion}

In this section, the numerical results are presented with the following assumptions: the equal power allocation strategy $\left(p_{k}=P / U\right)$ and noise effect $\left(\sigma_{k}=\sigma\right)$ for all users are the same [23]. Additionally, the AoD for each user is in a horizontal direction with the uniform distribution in the range $[-\pi, \pi]$. Furthermore, for each user the Ricean $K$-factor has uniform distribution in the range of $\left[K_{\min }, K_{\max }\right]$, where $K_{\min }=10 \mathrm{~dB}$ and $K_{\max }=30 \mathrm{~dB}$. Moreover, the number of users is increased

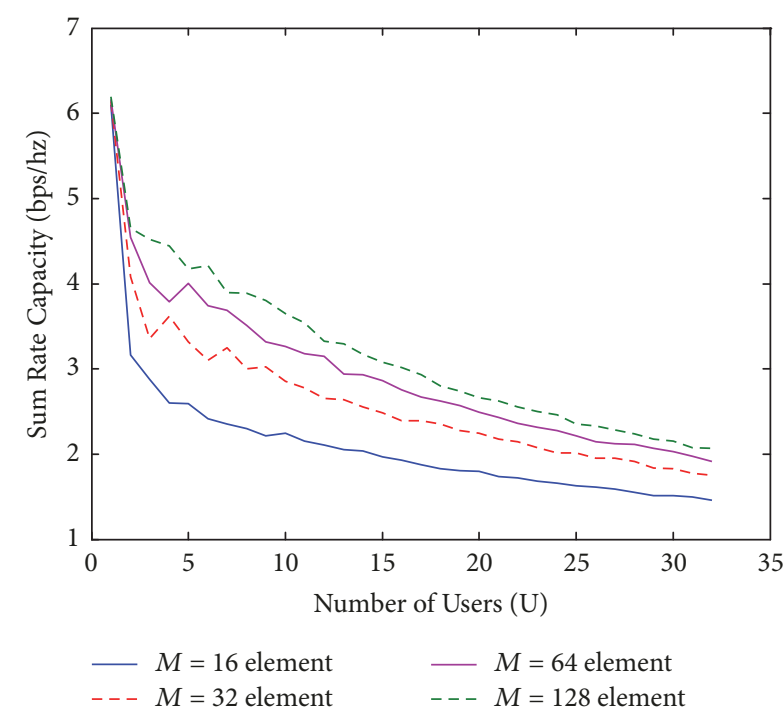

FIgURE 3: Sum-rate capacities for SE on the number of antenna elements.

up to $U=32$. For $\mathrm{SE}$ and $\mathrm{ZF}$ analysis $M=16,32,64$, and 128 are selected as the numbers of transmission antenna elements. The ergodic sum-rate capacities of SE and ZF are obtained in Figures 3 and 4. In Figure 3. It is observed that the average system capacity of SE is, respectively, directly and inversely proportional to the number of antenna elements and number of users. Moreover, when the number of users is small, the impact of $M$ on the SE capacity is more significant and conversely when the numbers are large, the impact is less significant. The SE achieves average capacities approximately in the range of $1.6-6.2 \mathrm{bps} / \mathrm{Hz}$. At maximum number of users $(U=32)$ with $M=16$, the corresponding SE sumrate capacity is less than $1.6 \mathrm{bps} / \mathrm{Hz}$. This is because each user faces CCI interference which is considered as sum of leakage signals from all other cochannel users. Referring to (17), it is validated that as the number of users increases, the overall contributed interference to the $k$ th user also increases. Therefore, the increase in the denominator in (17) causes a reduction in the sum-rate capacity per user resulting in an average lower system capacity.

In Figure 4, it is shown that the ergodic sum-rate capacities obtained by ZF are directly proportional to the number of antenna elements. By referring to (18) the interference from the other users is perfectly suppressed by the orthogonal beamforming for user channel and weight vectors. These vectors are orthogonal to each other based on the condition $\left|\mathbf{h}_{j} \mathbf{w}_{k}^{\mathrm{ZF}}\right|=0$ in which $k \neq j$.

Moreover, the number of antenna elements $M$ has much contribution on the average system capacity with ZF than SE as comparatively shown in Figures 4 and 3. The maximum achievable capacities are $105 \mathrm{bps} / \mathrm{Hz}$ and $6.2 \mathrm{bps} / \mathrm{Hz}$ for $\mathrm{ZF}$ and SE, respectively, because of the interference effects on SE.

Figure 5 shows the upper bound of the mean-rate capacity gap loss $\left(R^{\mathrm{ZF}}-R^{\mathrm{SE}}\right)$ between $\mathrm{ZF}$ and SE. Since the number of users tends to be large, the difference in the mean rate capacity is most likely the ZF capacity, because the capacity obtained 


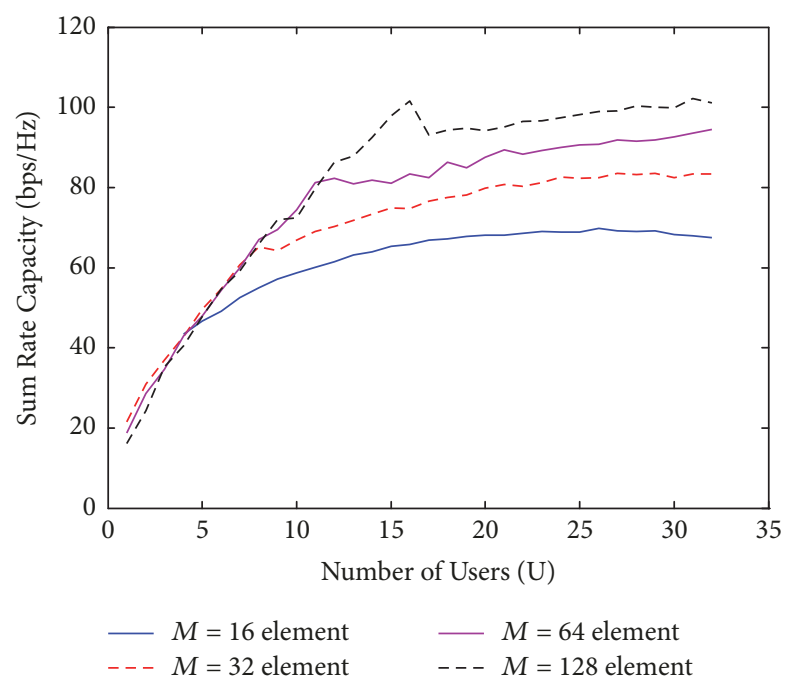

FIGURE 4: Achievable sum-rate capacities for ZF beamforming on the number of antenna elements.

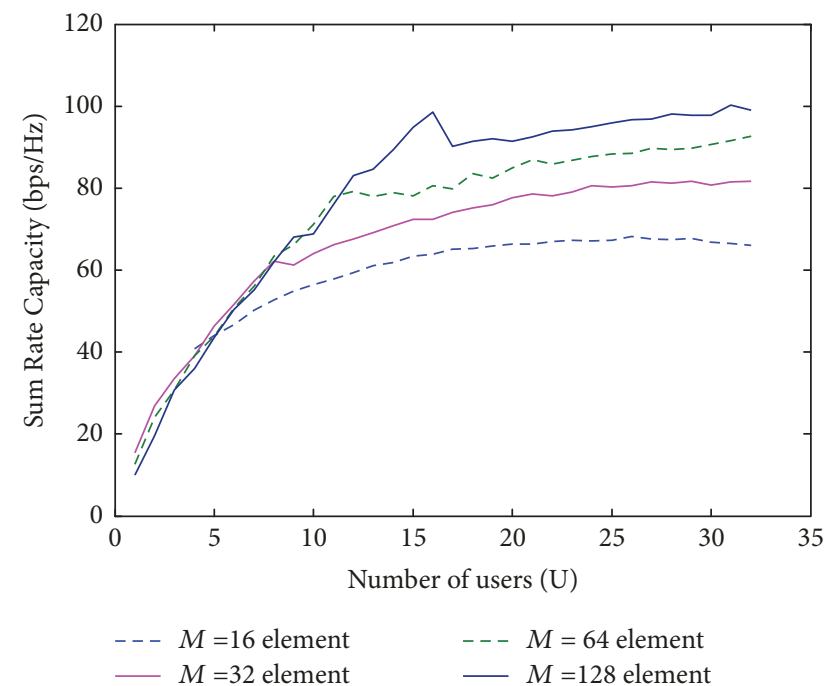

FIGURE 5: Mean gap loss capacity $\left(R^{\mathrm{ZF}}-R^{\mathrm{SE}}\right)$ which is the difference between ZF and SE capacities.

by $\mathrm{ZF}$ is close to 15 times that obtained by SE, which precisely affects the mean-gap loss. Nevertheless, when the number of users increases, the difference tends to be very big. It is explicit that when the number of users is large under any number of antenna elements, the achievable sum-rate capacity obtained by SE almost tends to be zero. However, the ergodic sum-rate capacity achieved by ZF positively increases with increase in the number of antenna elements and tends to a fixed level with large number of users. Meanwhile, the achievable sumrate capacity is not profoundly affected by the number of users.

As shown in Figures 3-5, the simulation results depict that the ZF seems ideal due to a perfect cancellation of CCI. However, in practice SE is dominant and realisable because it is challenging to ensure that the interference is perfectly cancelled by the orthogonal condition between the channel

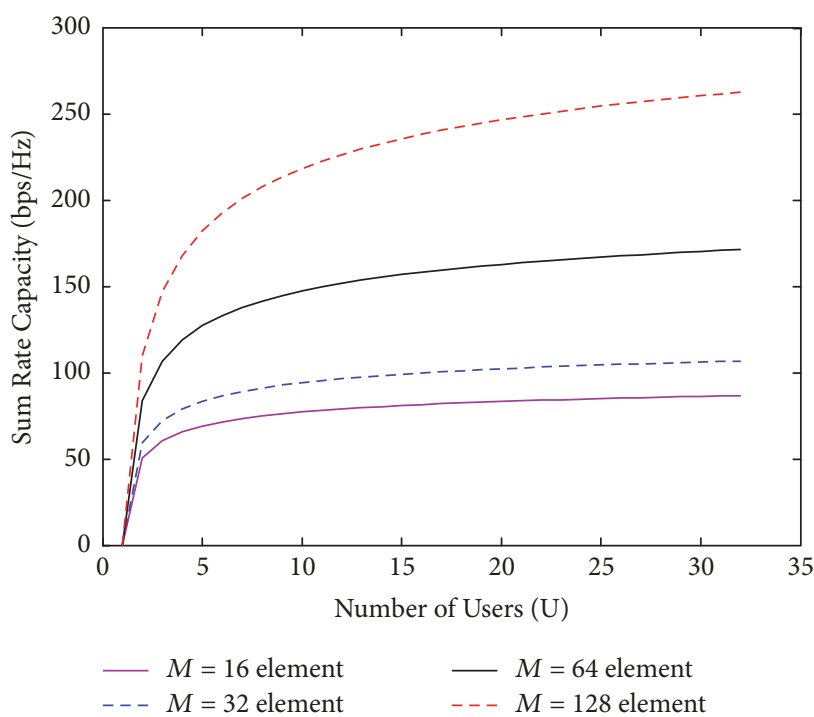

FIgURE 6: Achievable sum-rate capacities for DPC on the number of antenna elements.

vector and optimum weight vector. Thus, carefully focusing the beams alignment between the BS and the users a high system capacity can be achieved.

In Figure 6, the antenna elements $M=16,32,64$, and 128 are considered with number of users up to 32 to satisfy $M \geq U$ and show the effects of $M<U(M=16)$. The results show that the ergodic sum-rate capacity achieved by DPC is nonlinearly increased with the number of users. Moreover, if the number of users is fixed to 25 , at the number of antenna elements $16,32,64$, and 128 , respectively, the corresponding ergodic sum-rate capacities are 80, 105, 175, and $250 \mathrm{bps} / \mathrm{Hz}$, respectively. As the number of users is increased, the system capacity also increases. Thus, comparing the DPC with ZF and SE achieves much higher capacity with large number of antenna elements and users; hence it can provide multiuser diversity gain.

The results in Figures 7-9 are used to comparatively evaluate the proposed power iteration method in (38) and the SVD method in (30). In Figure 7, it is observed that the SLNR in the range $-10.5 \mathrm{~dB}$ to $37 \mathrm{~dB}$ achieves the system capacities in the range $5 \mathrm{bps} / \mathrm{Hz}$ to $11 \mathrm{bps} / \mathrm{Hz}$ for the proposed method. On the other hand, the SVD method has the SLNR in the range $-15 \mathrm{~dB}$ to $31 \mathrm{~dB}$ that achieves system capacities in the range $1 \mathrm{bps} / \mathrm{Hz}$ to $6 \mathrm{bps} / \mathrm{Hz}$. It is clear that the proposed method has the average throughput nearly two times that of SVD. The main reason is due to the efficiency of the weight vector obtained in the proposed method that is capable of minimising the CCI much more than in the SVD. It is expected that the system capacity can be enhanced in case the BS has more antenna elements but at a higher simulation time. Additionally, the SVD method has a drawback in finding the exact eigenvector corresponding to the dominant eigenvalue in which the eigenvalues are not always in an orderly way (ascending or descending), which limits the SVD performance. Figures 8 and 9 are the Cumulative Distribution Function (CDF) and Probability Density Function (PDF) of 

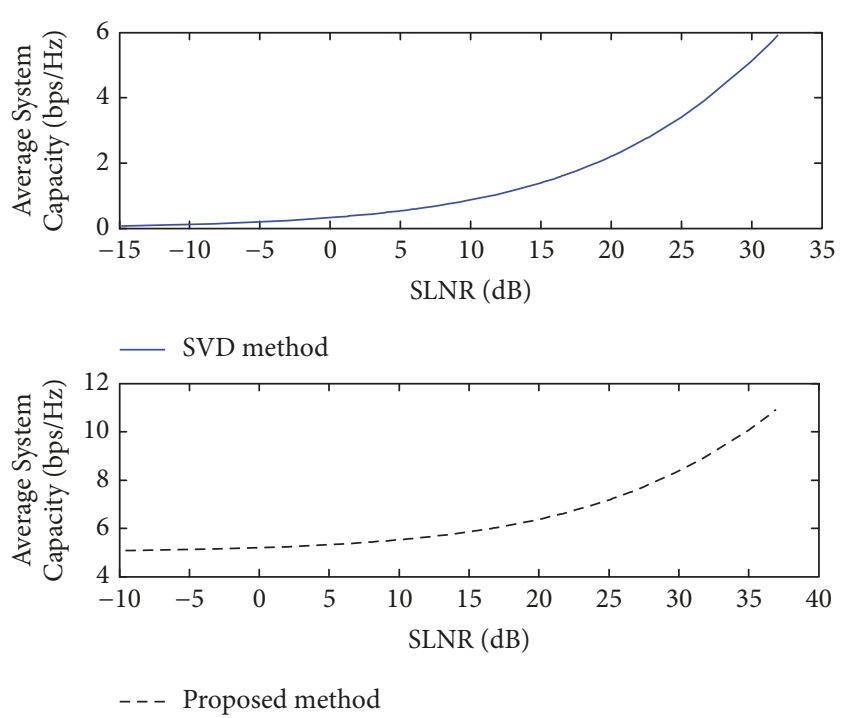

FIGURE 7: Sum-rate capacities for the proposed power iteration technique and SVD method.

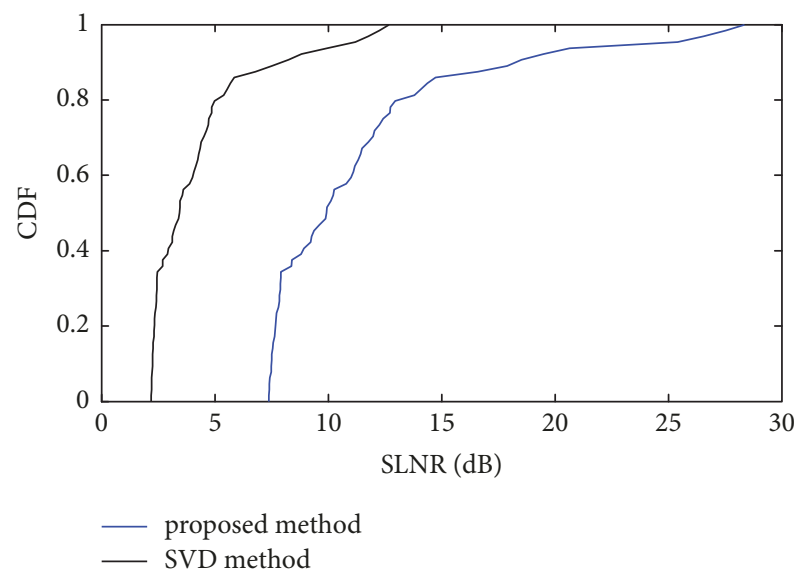

FIGURE 8: CDFs of the SLNRs for the proposed power iteration and SVD methods.

SLNR of the proposed and SVD methods, respectively. It is observed that the proposed method achieves a much better SLNRs than the SVD method.

\section{Conclusion}

In this paper, based on MU-MIMO with massive antennas, the ergodic capacity for linear sum-rate analysis including $\mathrm{SE}$ and ZF beamforming based on SLNR technique is investigated. In addition, the nonlinear sum-rate analysis using DPC in Ricean fading channels based on SLNR was undertaken. A new method is proposed to find an optimum beam weight vector by exploring the power iteration method using eigenvector approximation. The number of antenna elements is selected up to 128 dramatically increasing the system capacity. By way of simulation, the most significant dominant eigenvector to maximise SLNR as well as minimise the CCI is obtained. By comparing with the SVD method,

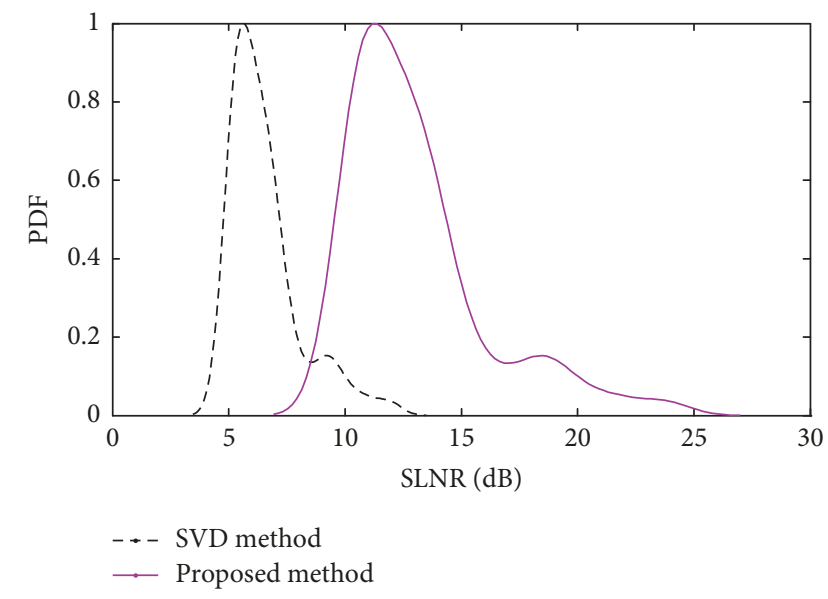

FIGURE 9: PDFs of the SLNRs for the proposed power iteration and SVD methods.

the proposed method achieves higher performance regarding mean achievable sum-rate capacity per user. The throughput of the proposed method is in the range of $5 \mathrm{bps} / \mathrm{Hz}-11 \mathrm{bps} / \mathrm{Hz}$, while that of SVD in the range of $1 \mathrm{bps} / \mathrm{Hz}-6 \mathrm{bps} / \mathrm{Hz}$. Therefore, the proposed method can provide significant system capacity enhancement. In future work, the proposed power iteration technique is recommended for a $5 \mathrm{G}$ MU massive antenna system.

\section{Appendix}

\section{A. Proof of the Algorithm}

As we know, matrix $\mathbf{A}$ has $n$ eigenvalues and $n$ corresponding eigenvectors $\mathbf{V}_{j}(j=1,2, \ldots, n)$ which are linearly independent and normalised and form a basis for $n$ dimensional space. Thus, let the initial vector $\mathbf{X}_{0}$ be written as the linear combination form as

$$
\mathbf{X}_{0}=b_{1} \mathbf{V}_{1}+b_{2} \mathbf{V}_{2}+\cdots+b_{n} \mathbf{V}_{n}
$$

Assume vector $\mathbf{X}_{0}=\left[\begin{array}{llll}x_{1} & x_{2} & \cdots & x_{n}\end{array}\right]^{T}$ is chosen in such a way that $b_{1} \neq 0$, and the elements of $\mathbf{X}_{0}$ are scaled so that $\max _{1 \leq j \leq n}\left\{\left|x_{j}\right|\right\}=1$. As the vectors $\left\{\mathbf{V}_{j}\right\}_{j=1}^{n}$ represent the eigenvectors of the matrix $\mathbf{A}$, the multiplication $\mathbf{A X}_{0}$ is followed by normalisation as follows:

$$
\begin{aligned}
\mathbf{Y}_{0} & =\mathbf{A} \mathbf{X}_{0}=\mathbf{A}\left(b_{1} \mathbf{V}_{1}+b_{2} \mathbf{V}_{2}+\cdots+b_{n} \mathbf{V}_{n}\right) \\
& =b_{1} \mathbf{A} \mathbf{V}_{1}+b_{2} \mathbf{A} \mathbf{V}_{2}+\cdots+b_{n} \mathbf{A} \mathbf{V}_{n} \\
& =b_{1} \lambda_{1} \mathbf{V}_{1}+b_{2} \lambda_{2} \mathbf{V}_{2}+\cdots+b_{n} \lambda_{n} \mathbf{V}_{n} \\
& =\lambda_{1}\left(b_{1} \mathbf{V}_{1}+b_{2}\left(\frac{\lambda_{2}}{\lambda_{1}}\right) \mathbf{V}_{2}+\cdots+b_{n}\left(\frac{\lambda_{n}}{\lambda_{1}}\right) \mathbf{V}_{n}\right) .
\end{aligned}
$$

Moreover, also we have

$\mathbf{X}_{1}$

$$
=\frac{\lambda_{1}}{c_{1}}\left(b_{1} \mathbf{V}_{1}+b_{2}\left(\frac{\lambda_{2}}{\lambda_{1}}\right) \mathbf{V}_{2}+\cdots+b_{n}\left(\frac{\lambda_{n}}{\lambda_{1}}\right) \mathbf{V}_{n}\right) .
$$


After $k$ iterations we arrive at

$$
\begin{aligned}
& \mathbf{Y}_{k-1}=\mathbf{A} \mathbf{X}_{k-1}=\mathbf{A} \frac{\lambda_{1}^{k-1}}{c_{1} c_{2} \cdots c_{k-1}}\left(b_{1} \mathbf{V}_{1}\right. \\
& \left.+b_{2}\left(\frac{\lambda_{2}}{\lambda_{1}}\right)^{k-1} \mathbf{V}_{2}+\cdots+b_{n}\left(\frac{\lambda_{2}}{\lambda_{1}}\right)^{k-1} \mathbf{V}_{n}\right) \\
& =\frac{\lambda_{1}^{k-1}}{c_{1} c_{2} \cdots c_{k-1}}\left(b_{1} \mathbf{A} \mathbf{V}_{1}+b_{2}\left(\frac{\lambda_{2}}{\lambda_{1}}\right)^{k-1} \mathbf{A} \mathbf{V}_{2}+\cdots\right. \\
& \left.+b_{n}\left(\frac{\lambda_{2}}{\lambda_{1}}\right)^{k-1} \mathbf{A} \mathbf{V}_{n}\right)=\frac{\lambda_{1}^{k-1}}{c_{1} c_{2} \cdots c_{k-1}}\left(b_{1} \lambda_{1} \mathbf{V}_{1}\right. \\
& \left.+b_{2}\left(\frac{\lambda_{2}}{\lambda_{1}}\right)^{k-1} \lambda_{2} \mathbf{V}_{2}+\cdots+b_{n}\left(\frac{\lambda_{2}}{\lambda_{1}}\right)^{k-1} \lambda_{n} \mathbf{V}_{n}\right) \\
& =\frac{\lambda_{1}^{k}}{c_{1} c_{2} \cdots c_{k-1}}\left(b_{1} \mathbf{V}_{1}+b_{2}\left(\frac{\lambda_{2}}{\lambda_{1}}\right)^{k} \mathbf{V}_{2}+\cdots\right. \\
& \left.+b_{n}\left(\frac{\lambda_{2}}{\lambda_{1}}\right)^{k} \mathbf{V}_{n}\right) .
\end{aligned}
$$

Furthermore, we have

$$
\begin{aligned}
\mathbf{X}_{k} & =\frac{\lambda_{1}^{k}}{c_{1} c_{2} \cdots c_{k}}\left(b_{1} \mathbf{V}_{1}+b_{2}\left(\frac{\lambda_{2}}{\lambda_{1}}\right)^{k-1} \mathbf{V}_{2}+\cdots\right. \\
& \left.+b_{n}\left(\frac{\lambda_{2}}{\lambda_{1}}\right)^{k-1} \mathbf{V}_{n}\right)
\end{aligned}
$$

Since we assumed that $\left|\lambda_{j}\right| /\left|\lambda_{1}\right|<1$ for $j=2,3, \ldots, n$, then we have

$$
\lim _{k \rightarrow \infty} b_{j}\left(\frac{\lambda_{j}}{\lambda_{1}}\right) \mathbf{V}_{j}=0, \quad \text { for } j=2,3, \ldots, n .
$$

Hence it follows that

$$
\lim _{k \rightarrow \infty} \mathbf{X}_{k}=\lim _{k \rightarrow \infty} \frac{b_{1} \lambda_{1}^{k}}{c_{1} c_{2} \cdots c_{k}} \mathbf{V}_{1}
$$

We need both vectors $\mathbf{X}_{k}$ and $\mathbf{V}_{1}$ to be normalised and their most significant component is 1 .

$$
\lim _{k \rightarrow \infty} \frac{b_{1} \lambda_{1}^{k}}{c_{1} c_{2} \cdots c_{k}}=1 .
$$

\section{B. Proof of the Convergence}

For the sequence of vectors $\left\{\mathbf{X}_{k}\right\}$ which converges to the dominant eigenvector:

$$
\lim _{x \rightarrow \infty} \mathbf{X}_{k}=\mathbf{V}_{1} \text {. }
$$

By substituting $k$ with $k-1$, it yields

$$
\lim _{k \rightarrow \infty} \frac{b_{1} \lambda_{1}^{k-1}}{c_{1} c_{2} \cdots c_{k-1}}=1 .
$$

By merging both into (A.8), we get

$$
\lim _{x \rightarrow \infty} \frac{\lambda_{1}}{c_{k}}=\lim _{x \rightarrow \infty} \frac{b_{1} \lambda_{1}^{k} / c_{1} c_{2} \cdots c_{k}}{b_{1} \lambda_{1}^{k-1} / c_{1} c_{2} \cdots c_{k-1}}=\frac{1}{1}=1 .
$$

Therefore, the sequences of the constants $\left\{c_{k}\right\}$ converge to the dominant eigenvalue as

$$
\lim _{x \rightarrow \infty} c_{k}=\lambda_{1}
$$

\section{Data Availability}

The data used to support the findings of this study are available from the corresponding author upon request.

\section{Conflicts of Interest}

The authors declare that there are no conflicts of interest regarding the publication of this paper.

\section{Acknowledgments}

This work is supported by Key program of Beijing Municipal Natural Science Foundation with no. 17L20052 and also supported by National Key Laboratory of Electromagnetic Environment, China Research Institute of Radiowave Propagation under Grant no. 201600012, and the National Nature Science Foundation of China (NSFC) under Grant no. 61771194.

\section{References}

[1] M. S. Islam, M. Kamruzzaman, T. Jessy, M. S. Zahan, and M. S. Hassan, "Performance analysis of massive MIMO for $5 \mathrm{G}$ wireless communication systems," in Proceedings of the 2016 IEEE International Conference on Computing, Communication and Automation, ICCCA 2016, pp. 1579-1583, April 2016.

[2] R. W. Heath, N. Gonzalez-Prelcic, S. Rangan, W. Roh, and A. Sayeed, "Introduction to the special issue on signal processing for millimeter wave wireless communications," IEEE Journal of Selected Topics in Signal Processing, vol. 10, no. 3, pp. 433-435, 2016.

[3] H. Shen, W. Xu, A. L. Swindlehurst, and C. Zhao, "Transmitter optimization for per-antenna power constrained multi-antenna downlinks: an SLNR maximization methodology," IEEE Transactions on Signal Processing, vol. 64, no. 10, pp. 2712-2725, 2016.

[4] M. Sadek, A. Tarighat, and A. H. Sayed, "A lakage-based precoding scheme for downlink multi-user MIMO channels," IEEE Transactions on Wireless Communications, vol. 6, no. 5, pp. 1711-1721, 2007.

[5] M. Vu and A. Paulraj, "Linear precoding for MIMO wireless correlated channels with non-zero means: $\mathrm{K}$ factor analysis, extension to non-orthogonal STBC," in Proceedings of the (ICASSP '05). IEEE International Conference on Acoustics, Speech, and Signal Processing, 2005., pp. 1113-1116, Philadelphia, PA, USA, 2005.

[6] C. Zhang, Z. Lu, Y. Huang, J. Zhang, and L. Yang, "Statistical beamforming for FDD massive MIMO downlink systems," in Proceedings of the IEEE/CIC International Conference on Communications in China, ICCC 2015, November 2015.

[7] A. Tarighat, M. Sadek, and A. H. Sayed, "A multi user beamforming scheme for downlink mimo channels based on 
maximizing signal-to-leakage ratios," in Proceedings of the IEEE International Conference on Acoustics, Speech, and Signal Processing (ICASSP '05), vol. 3, pp. 1129-1132, IEEE, March 2005.

[8] D. Zhang, Z. Zhou, C. Xu, Y. Zhang, J. Rodriguez, and T. Sato, "Capacity analysis of NOMA with mmWave massive MIMO systems," IEEE Journal on Selected Areas in Communications, vol. 35, no. 7, pp. 1606-1618, 2017.

[9] J. Choi, "Minimum power multicast beamforming with superposition coding for multiresolution broadcast and application to NOMA systems," IEEE Transactions on Communications, vol. 63, no. 3, pp. 791-800, 2015.

[10] S. Park, J. Park, A. Yazdan, and R. W. Heath, "Optimal user loading in massive MIMO systems with regularized zero forcing precoding," IEEE Wireless Communications Letters, vol. 6, no. 1, pp. 118-121, 2017.

[11] S. Jin, W. Tan, M. Matthaiou, J. Wang, and K.-K. Wong, "Statistical eigenmode transmission for the MU-MIMO downlink in rician fading," IEEE Transactions on Wireless Communications, vol. 14, no. 12, pp. 6650-6663, 2015.

[12] X. Li, S. Jin, H. A. Suraweera, J. Hou, and X. Gao, "Statistical 3D beamforming for large-scale MIMO downlink systems over rician fading channels," IEEE Transactions on Communications, vol. 64, no. 4, pp. 1529-1543, 2016.

[13] I. Boukhedimi, A. Kammoun, and M.-S. Alouini, "Coordinated SLNR based precoding in large-scale heterogeneous networks," IEEE Journal of Selected Topics in Signal Processing, vol. 11, no. 3, pp. 534-548, 2017.

[14] X. Wu, W. Zhao, X. Sha, F. Labeau, and Y. Wang, "SLNR beamforming based iterative power allocation in TD-LTE-A downlink," in Proceedings of the 11th International Wireless Communications and Mobile Computing Conference, IWCMC 2015, pp. 84-89, August 2015.

[15] L. Liu, D. W. Matolak, C. Tao, and Y. Li, "Analysis of an upper bound on the effects of large scale attenuation on uplink transmission performance for massive MIMO systems," IEEE Access, vol. 5, pp. 4285-4297, 2017.

[16] C. Zhang, Y. Huang, Y. Jing, S. Jin, and L. Yang, "Sum-rate analysis for massive MIMO downlink with joint statistical beamforming and user scheduling," IEEE Transactions on Wireless Communications, vol. 16, no. 4, pp. 2181-2194, 2017.

[17] X. Zhao, A. M. Abdo, C. Xu, S. Geng, J. Zhang, and I. Memon, "Dimension reduction of channel correlation matrix using CUR-decomposition technique for 3-D massive antenna system," IEEE Access, vol. 6, pp. 3031-3039, 2018.

[18] O. E. Ayach, S. Rajagopal, S. Abu-Surra, Z. Pi, and R. W. Heath, "Spatially sparse precoding in millimeter wave MIMO systems," IEEE Transactions on Wireless Communications, vol. 13, no. 3, pp. 1499-1513, 2014.

[19] T. Yoo and A. Goldsmith, "Optimality of zero-forcing beamforming with multiuser diversity," in Proceedings of the 2005 IEEE International Conference on Communications, ICC 2005, pp. 542-546, May 2005.

[20] M. Sharif and B. Hassibi, "A comparison of time-sharing, DPC, and beamforming for MIMO broadcast channels with many users," IEEE Transactions on Communications, vol. 55, no. 1, pp. $11-15,2007$.

[21] J. Wang, X. Xie, and Q. Zhang, "A way to reduce ICI of multiuser MIMO-OFDM system with precoding," in Proceedings of the International Conference on Advanced Computer Control, ICACC 2009, pp. 134-137, January 2009.

[22] Y. Saad, Numerical Methods for Large Eigenvalue Problems, Classics in Applied Mathematics, Society for Industrial and
Applied Mathematics (SIAM), Philadelphia, PA, USA, 2nd edition, 2011.

[23] A. M. Abdo, X. Zhao, R. Zhang, Y. Zhang, K. Eguchi, and T. Chen, "Codebook metrics evaluation for millimeter wave communications by antenna array response and signal to noise ratio," ITM Web of Conferences, vol. 17, p. 03011, 2018. 


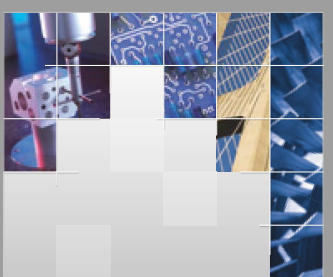

\section{Enfincering}
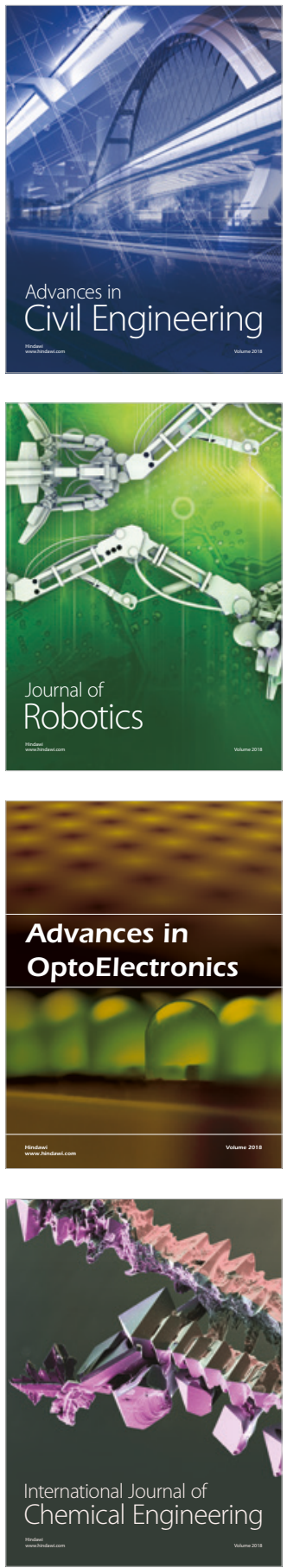

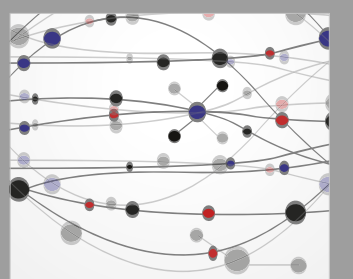

\section{Rotating \\ Machinery}

The Scientific World Journal

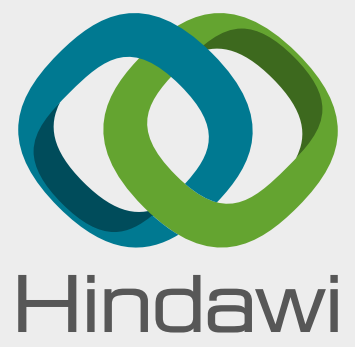

Submit your manuscripts at

www.hindawi.com
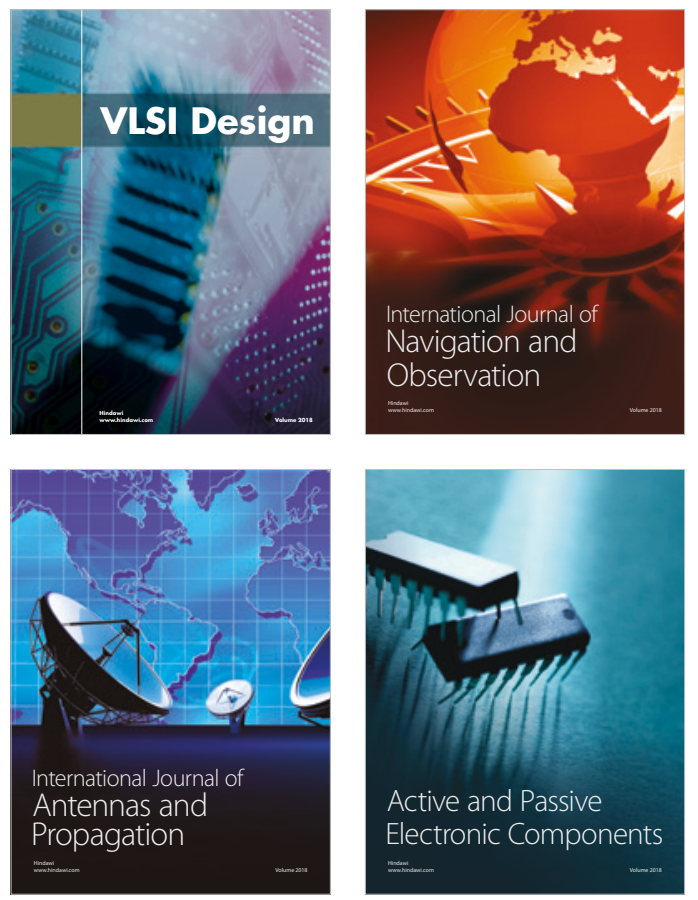
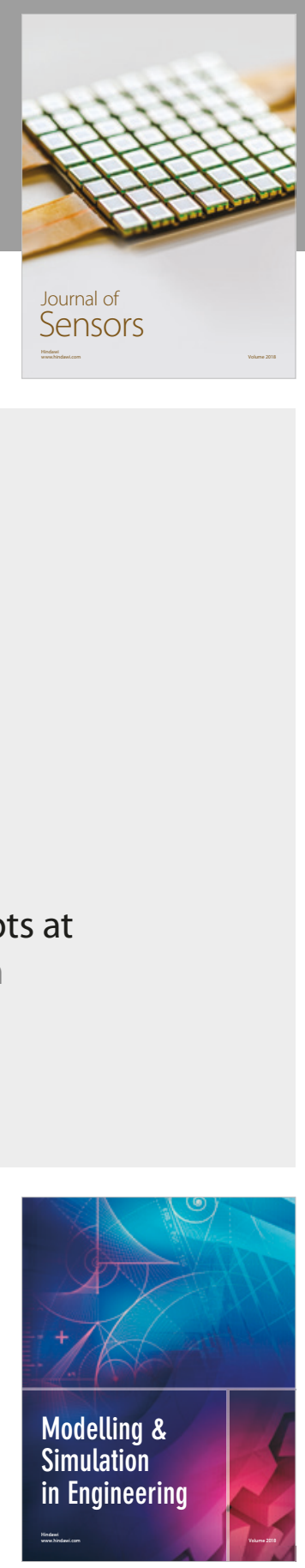

\section{Advances \\ Multimedia}
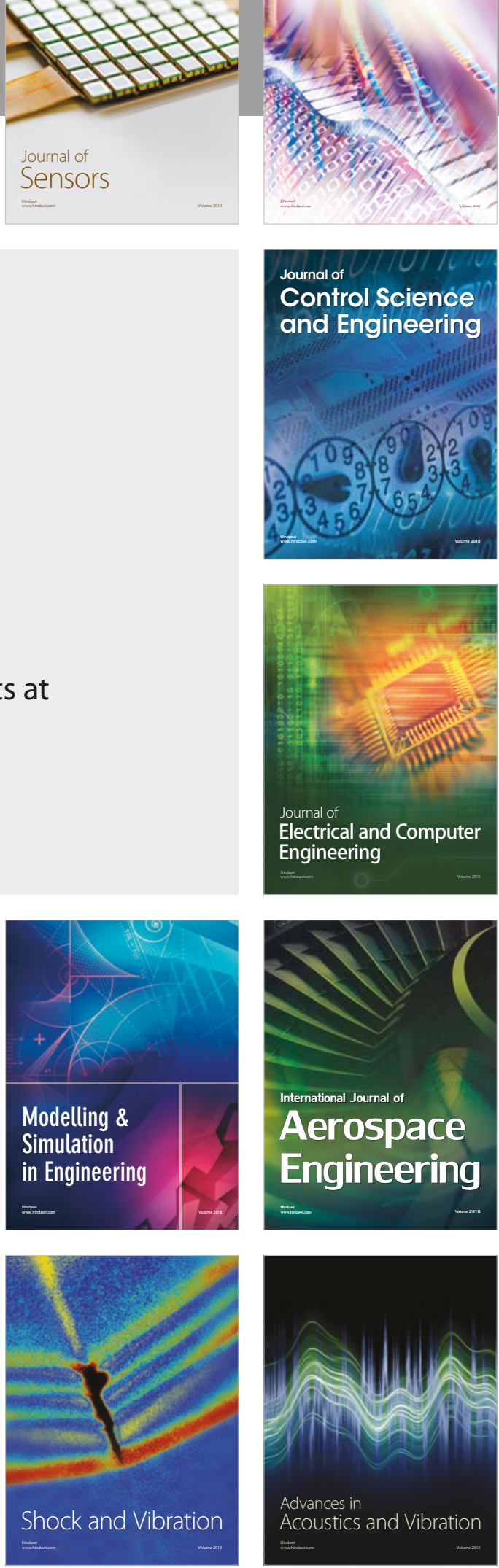